\title{
ARROW-HAHN ECONOMIC MODELS WITH WEAKENED CONDITIONS OF CONTINUITY
}

\author{
INESE BULA \\ Department of Mathematics, University of Latvia \\ Rainis boulv. 19, Rìga, LV-1586, Latvia \\ E-mail: ibula@lanet.lv \\ DACE RIKA \\ Department of Mathematics, University of Latvia \\ Rainis boulv. 19, Rīga, LV-1586, Latvia \\ E-mail:dace.rika@vidm.gov.lv
}

\begin{abstract}
In this article we give descriptions of some economic models that are based on Arrow-Hahn economic model. Finally we consider a model with two major assumptions: first, there is discontinuous excess demand function and, second, if price goes to zero, then it is possible that excess demand may approach infinity. For this last new economic model the existence of quasi-equilibrium is proved.
\end{abstract}

1. Introduction. One of the research directions of microeconomics is finding such conditions and assumptions that there exists an equilibrium in a given economic model. The basic assumption in equilibrium economic models is the continuity of the supply function, demand function, excess demand function or multi function involved (see, for example, [17], [2], [9], [3], or textbooks on microeconomics, for example, [16], [13], [15]). We consider situations with weakened conditions of continuity.

First of all in section 2 we give the description of classical Arrow-Hahn model with continuous excess demand function ([3], chapter 1). In section 3 we consider the Arrow-Hahn model with weakened condition of continuity: if the price is 0 then the value of the excess demand function can tend to $+\infty$ (see [3] too). We will weaken the condition of continuity even more, by considering the situation with discontinuous excess demand function.

2000 Mathematics Subject Classification: Primary 91B50; Secondary 26A15.

Key words and phrases: Arrow-Hahn economic model, economic equilibrium, quasi-equilibrium, continuous function, discontinuous function.

Research of the first author supported by grant 01.0527 of the Latvian Academy of Science. The paper is in final form and no version of it will be published elsewhere. 
Economic literature analyses the situations with continuous excess demand function. The classical mathematical theory used in models of equilibrium economics is theory of continuity (for example, Brouwer or Kakutani theorems for continuous functions). These mathematical tools are convenient to use and therefore they are considered most often since there are no better tools. We will apply $w$-discontinuous functions $([4],[5],[6])$ to the study of economics. The excess demand function is continuous in a stable economic situation but it may not be continuous if price changes fast (i.e., very high increase of inflation or changes in the political system); also the economic agents can suddenly change their decision regarding to a particular good because of marketing or advertising. In principle the considered cases are exceptional situations, extreme cases, but they exist and influence the economic life. We note that demand and supply functions are discontinuous for piece-goods like airplanes, hydroelectric stations, cars and even tables, shoes, shirts and other goods being sold by unit or piece and assuming that nobody buys for example, $1 / 2$ of the car. This is because demand or supply of such goods is measured in integers. Therefore in section 4 we introduce the $w$-discontinuous functions and their properties, in section 5 we give a short review of the Arrow-Hahn model with $w$-discontinuous excess demand function (see [6]). Finally we consider the new model with basic assumptions of sections 5 and 3 together. We use another proof technique based on results of section 5 and we prove a quasi-equilibrium theorem. We conclude that this quasi-equilibrium is bounded.

2. The Arrow-Hahn economic model. We give the description of an economic model considered by Arrow and Hahn in [3].

Let there be $n(n \in \mathbf{N})$ different goods (commodities) on the market and a finite number of economic agents: households and firms.

Let $x_{h i}$ be the quantity of good $i$ which is needed to the household $h$. The summation over all households will be indicated by $x_{i}=\sum_{h} x_{h i}$ - the total demand of good $i, i=$ $1, \ldots, n$.

The quantity of good $i$ that is supplied by the firm $f$ will be denoted by $y_{f i}$. The summation over all firms will be indicated by $y_{i}=\sum_{f} y_{f i}-$ the supply of good $i, i=$ $1, \ldots, n$.

The initially available amount (or resources) of good $i$ in all households will be denoted by $\overline{x_{i}}$. Note that $\overline{x_{i}}$ must be non-negative.

Market equilibrium describes the economic situation that the total demand of each good in the economy is satisfied by its total supply. This fact is obviously expressed by saying that the difference between the total demand of each good and its total supply is less than or equal to zero. The total supply of good $i$ is understood as the sum of the supply of the good $i$ and the quantity of $i$ which is already available, i.e., the total supply of the good $i$ equals $y_{i}+\overline{x_{i}}$. The excess demand of good $i$ is then defined as $x_{i}-y_{i}-\overline{x_{i}}$, $i=1, \ldots, n$.

Further on we frequently make use of the natural order in $\mathbf{R}^{n}$ introduced by the cone

$$
\mathbf{R}_{+}^{n}=\left\{x=\left(x_{1}, \ldots, x_{n}\right) \in \mathbf{R}^{n} \mid x_{i} \geq 0, i=1, \ldots, n\right\},
$$

i.e., for two vectors $x=\left(x_{1}, \ldots, x_{n}\right), y=\left(y_{1}, \ldots, y_{n}\right)$ we write $x \leq y$ iff $x_{i} \leq y_{i}$ for all 
$i=1, \ldots, n$, we write $x<y$ iff $x \leq y$ and $x_{i_{0}}<y_{i_{0}}$ for at least one index $i_{0}$. The norm we will use in the space $\mathbf{R}^{n}$ is defined as

$$
\|x\|=\sum_{i=1}^{n}\left|x_{i}\right|, x=\left(x_{1}, \ldots, x_{n}\right) \in \mathbf{R}^{n} .
$$

This norm is equivalent to the Euclidean norm which is introduced by means of the scalar product $\langle x, y\rangle=\sum_{i=1}^{n} x_{i} y_{i}$. Note that in economic publications the scalar product of two vectors $x, y \in \mathbf{R}^{n}$ is usually written as $x y$.

For the standard economic model the following four assumptions have to be met.

Assumption F. Let $p=\left(p_{1}, \ldots, p_{n}\right)$ be an $n$-dimensional price vector with the prices $p_{i}$ for one unit of the good $i, i=1,2, \ldots, n$. For any $p$ let the excess demand for $i$ be characterized by a unique number $z_{i}(p)$ and so the unique vector $z(p)=\left(z_{1}(p), \ldots, z_{n}(p)\right)$ the excess demand function with excess demand functions for $i$ as components $(i=$ $1,2, \ldots, n)$ - is well defined.

Assumption H. $\quad z(p)=z(\lambda p), \quad \forall p>\mathbf{0}$ and $\lambda>0$.

Assumption $\mathrm{H}$ asserts that $z$ is a homogeneous vector-function of degree zero. Economically this means that the value of the excess demand function does not depend on the price system if the latter is changed for all the goods simultaneously by the same portion.

From Assumption $\mathrm{H}$ it follows that prices can be normalized (see [3], p. 20 or [8], p. 10). If for some price $p$ one has $z(p)=0$ then $z(\lambda p)=0$ for all prices of the ray $\{\lambda p \mid \lambda>0\}$. Therefore, further on we consider only prices from the $(n-1)$-dimensional simplex of $\mathbf{R}^{n}$

$$
S_{n}=\left\{p=\left(p_{1}, p_{2}, \ldots, p_{n}\right) \mid p_{i} \geq 0 \text { and } \sum_{i=1}^{n} p_{i}=1\right\} .
$$

We rule out the situations when all the prices are zero or some of them are negative. Note that $S_{n}$ is a compact and convex set in the space $\mathbf{R}^{n}$.

Assumption W or Walras' LaW. $\quad p z(p)=0, \quad \forall p \in S_{n}$.

Walras' Law can be regarded as an attempt to have a model sufficiently truly reflecting rationally motivated activities of economic agents. According to Walras' Law all the firms and all the households both spend their financial resources completely ([8]).

Assumption C. The excess demand function $z$ is continuous on its domain of definition $S_{n}$.

It means that a small change of a price system will imply only a small change in the excess demand. As a consequence from continuity of $z$, the standard model can be used only for the description of economies with continuous excess demand functions.

Definition 2.1. A price $p^{*} \in S_{n}$ is called an equilibrium (price) if $z\left(p^{*}\right) \leq 0$.

If $p^{*}$ is an equilibrium price then $\sum_{i=1}^{n} z_{i}\left(p^{*}\right) \leq 0$.

For the standard model of an economy with a finite number of goods and agents such prices always exist as is proved in the following theorem. 
THEOREM 2.1 ([3]). If an economy with a finite number of goods and agents satisfies Assumptions $F, H, W$ and $C$, then there exists an equilibrium.

3. The Arrow-Hahn economic model with weakened continuity condition. In this section we continue description of another Arrow-Hahn model with weakened continuity assumption ([3], p. 29-33).

Assumption $\mathrm{C}$ implies that the demand for free goods is bounded. "We shall want to weaken this restriction for several reasons: it is not unreasonable that demand for at least some goods might approach infinity as the price approaches zero; the non-satiation hypothesis that underlies Walras' law it at least partly inconsistent with satiation in any single good; the assumption that all goods are gross substitutes, ..., implies that demands may approach infinity as prices go to zero." (We cite [3], p. 29.)

Therefore we will assume that the sum of excess demands approaches infinity whenever excess demand is undefined. First we introduce another assumption.

Assumption B. There exists a positive number $B$ such that for all $p \in S_{n}$ : $z_{i}(p)>-B$ for all $i=1,2, \ldots, n$, i.e., $z(p)$ is bounded from below.

Now we introduce new continuity assumption.

Assumption C'. The excess demand function $z$ is defined for every $p \in S_{n}$ such that $p_{i}>0, i=1,2, \ldots, n$, and possibly for other $p$ and is continuous whenever defined. If $z$ is not defined for $p^{0}$, then

$$
\lim _{p \rightarrow p^{0}} \sum_{i=1}^{n} z_{i}(p)=+\infty .
$$

It is possible to show that the previous Theorem 2.1 can be modified so that an equilibrium exist.

THEOREM 3.1 ([3]). If an economy with a finite number of goods and agents satisfies Assumptions $F, H, W, B$ and $C^{\prime}$ then there exists an equilibrium.

We remark that it follows from the proof of Theorem 3.1 that the equilibrium $p^{*}$ is a price vector from $S_{n}$ at which $z$ is defined.

4. w-discontinuous mappings and their properties. A class of discontinuous mappings is defined as follows. Let $(X, d)$ and $(Y, \varrho)$ be two metric spaces and $w$ a positive number.

Definition 4.1. A mapping $f: X \rightarrow Y$ is said to be $w$-discontinuous at the point $x_{0} \in X$ if for every $\varepsilon>0$ there exists $\delta$ such that whenever $x \in X$ and $d\left(x_{0}, x\right)<\delta$ follows that $\varrho\left(f\left(x_{0}\right), f(x)\right)<\varepsilon+w$.

The constant $w$ may not be the best possible (smallest) one. Very often, especially in economic applications, there is known only a rough upper estimation for the "jump".

A mapping $f$ is called $w$-discontinuous in $X$ if it is $w$-discontinuous at all points of $X$.

The notion of $w$-discontinuous maps is not new. It is already found in [14] as the concept of oscillation or as continuity defect in [7]. The notion of $w$-discontinuity was introduced by the first author in [4]. 
ExAmple 4.1. The usual Dirichlet function on $\mathbf{R}$ and also the generalized Dirichlet function $f: \mathbf{R}^{n} \rightarrow\{0,1\}$, defined for all $x=\left(x_{1}, x_{2}, \ldots, x_{n}\right) \in \mathbf{R}^{n}$ by

$$
f(x)=\left\{\begin{array}{l}
1, \text { if all components } x_{i} \in \mathbf{Q}, \\
0, \text { if there exists } i_{0} \text { such that } x_{i_{0}} \in \mathbf{R} \backslash \mathbf{Q},
\end{array}\right.
$$

are 1-discontinuous (and for any $w \geq 1$ also $w$-discontinuous).

If $X, Y, V$ are real normed vector spaces the following properties of $w$-discontinuous mappings are established (simmilar as for continuous mappings). For proofs and other comments see $[6]$.

Proposition 4.1. Let be $f_{i}: X \rightarrow Y, \alpha_{i} \in \mathbf{R}, i=1, \ldots, k$ and $g=\alpha_{1} f_{1}+\cdots+\alpha_{k} f_{k}$. Suppose $w_{i}>0$ and that $f_{i}$ is $w_{i}$-discontinuous on the set $X$ for each $i=1, \ldots, k$. Then $g=\alpha_{1} f_{1}+\cdots+\alpha_{k} f_{k}$ is a $\left|\alpha_{1}\right| w_{1}+\cdots+\left|\alpha_{k}\right| w_{k}$-discontinuous mapping.

From Definition 4.1 for $w=0$ immediately follows that the 0-discontinuous mappings are exactly the continuous ones.

Corollary 4.1. Suppose that $f, g: X \rightarrow Y, f$ is $w^{\prime}$-discontinuous and $g$ is $w^{\prime \prime}$-discontinuous. Then $f+g$ and $f-g$ are $w^{\prime}+w^{\prime \prime}$-discontinuous mappings. In particular, if one of the mappings ( $f$ or $g$ ) is continuous, then $f \pm g$ are $w^{\prime}$-discontinuous (or $w^{\prime \prime}$ discontinuous).

Corollary 4.2. If $f: X \rightarrow Y$ is $w$-discontinuous and $c$ is a constant then $c \cdot f$ is a $|c|$ w-discontinuous mapping.

In order to consider the product of mappings we need the notion of the product in a normed space.

Definition $4.2([12])$. Let $X, Y, Z$ be real normed vector spaces. A mapping $\pi: X \times Y \rightarrow Z$ is called a product if it satisfies the following conditions: for all $a, b \in$ $X, u, v \in Y$ and $\lambda \in \mathbf{R}$ one has

1. $\pi((a+b, v))=\pi((a, v))+\pi((b, v))$

2. $\pi((a, u+v))=\pi((a, u))+\pi((a, v))$

3. $\pi((\lambda a, u))=\lambda \pi((a, u))=\pi((a, \lambda u))$

4. $\|\pi((a, u))\|_{Z} \leq\|a\|_{X}\|u\|_{Y}$.

A simple example is given by $X=Y=\mathbf{R}^{n}, Z=\mathbf{R}$ and $\pi((x, y))=\langle x, y\rangle$, the scalar product in $\mathbf{R}^{n}$, i.e., $\langle x, y\rangle=\sum_{i=1}^{n} x_{i} y_{i}$.

Let $V, X, Y, Z$ be real normed vector spaces and let $\pi: X \times Y \rightarrow Z$ be a product. The product of the mappings $f: \operatorname{dom} f \subseteq V \rightarrow X$ and $g: \operatorname{dom} g \subseteq V \rightarrow Y$ is understood pointwise, i.e.,

$$
(f \cdot g)(v)=\pi(f(v), g(v)), \quad \forall v \in \operatorname{dom} f \cap \operatorname{dom} g,
$$

where $\operatorname{dom} f, \operatorname{dom} g \subseteq V$.

Proposition 4.2. Suppose that $f: \operatorname{dom} f \rightarrow X$ is $w^{\prime}$-discontinuous and $g: \operatorname{dom} g \rightarrow Y$ is $w^{\prime \prime}$-discontinuous on $\operatorname{dom} f \cap \operatorname{dom} g$. Then $f \cdot g$ is $a\left(w^{\prime} w^{\prime \prime}+w^{\prime}\left\|g\left(x_{0}\right)\right\|_{Y}+w^{\prime \prime}\left\|f\left(x_{0}\right)\right\|_{X}\right)$ discontinuous mapping at every point $x_{0} \in \operatorname{dom} f \cap \operatorname{dom} g$. 
Corollary 4.3. If $f: V \rightarrow X$ is w-discontinuous and $g: V \rightarrow Y$ is continuous then $f \cdot g$ is a $\left\|g\left(x_{0}\right)\right\|_{Y} w$-discontinuous mapping at every point $x_{0} \in V$.

For the division we reconcile with simplified situation, where $(X, d)$ is again a metric space.

Proposition 4.3. Let the function $f: X \rightarrow \mathbf{R}$ be $w$-discontinuous at the point $x_{0}$ and $f\left(x_{0}\right) \neq 0$. If there exists a neighbourhood $U$ of $x_{0}$ and a number $\alpha_{0}>0$ such that $|f(x)| \geq \alpha_{0}$ for all $x \in U$ then the function $\frac{1}{f}$ is $\frac{w}{\alpha_{0}\left|f\left(x_{0}\right)\right|}$-discontinuous at $x_{0}$.

As a special case we get

Corollary 4.4. If $f: X \rightarrow\left[1,+\infty\left[\right.\right.$ is $w$-discontinuous then $\frac{1}{f}$ is a $\frac{w}{f\left(x_{0}\right)}$-discontinuous mapping for every point $x_{0} \in X$

If the domain of definition for a continuous mapping is compact, then its range is also compact and, in particular, bounded. The boundedness of the most functions used in economic models seems to be indispensable in studying such models. The boundedness of the range is guaranteed for $w$-discontinuous mappings as well, however, compactness may not hold.

Example 4.2. Define $f:[0 ; 1] \rightarrow[0 ; 1]$ as

$$
f(x)=\left\{\begin{array}{l}
1, \text { if } x=0, \\
x, \text { if } x \in(0,1] .
\end{array}\right.
$$

The function $f$ is 1-discontinuous and its range $(0,1]$ is bounded, but not compact.

Theorem 4.1. Suppose that $A \subset X$ is compact and let $f: A \rightarrow X$ be $w$-discontinuous. Then $f(A)$ is bounded.

The following essential result is proved by O. Zaytsev in [18] and can be considered as a generalization of the Bohl-Brouwer-Schauder fixed point theorem for $w$-discontinuous mappings.

TheOREM 4.2. Let $K$ be a nonempty, compact and convex subset in a normed vector space $X$. For every $w$-discontinuous mapping $f: K \rightarrow K(w>0)$ there exists a point $x^{*} \in K$ such that $\left\|x^{*}-f\left(x^{*}\right)\right\| \leq w$.

5. Economic models with $w$-discontinuous excess demand functions. In this section we shortly analyse a model of an economy with $w$-discontinuous excess demand functions. For wider discussion see [6].

For the economies considered we keep the first two assumptions from the Arrow-Hahn model in Section 2 and change the last two as follows.

Assumption DC. The excess demand function $z$ is $w$-discontinuous on its domain of definition $S_{n}$.

It is quite natural that for every price vector $p \in S_{n}$ there exists at least one good $i$ with the price $p_{i}>0$ and such that the demand for it is satisfied, i.e., $z_{i}(p) \leq 0$. Therefore for each $p \in S_{n}$ the inequality $\gamma_{p}=\sum_{z_{i}(p) \leq 0} p_{i}>0$ is satisfied. Our next assumption requires the existence of a uniform lower bound for the sums $\sum_{z_{i}(p) \leq 0} p_{i}$ for all $p \in S_{n}$. 
Assumption $\Gamma \cdot \gamma=\inf _{p \in S_{n}} \gamma_{p}>0$.

This Assumption $\Gamma$ is independent of Walras' Law (see [6]). This means it is possible to find four different situations that

1) Walras' Law holds but not $\Gamma$,

2) $\Gamma$ holds but not Walras' Law (see Example 7.1),

3) $\Gamma$ and Walras' Law both hold (see Example 7.2),

4) neither Walras' Law nor $\Gamma$ holds.

We replace Assumption W or Walras' Law with Assumption $\Gamma$.

It would be hard to determine why an equilibrium exists in our model, but it will be possible if we can estimate the unsatisfied aggregate demand. This leads to the concept of quasi- or $k$-equilibrium.

Definition 5.1. Let $k>0$. A price vector $p^{*} \in S_{n}$ is called a $k$-equilibrium if it satisfies the condition

$$
\sum_{z_{i}\left(p^{*}\right)>0} z_{i}\left(p^{*}\right) \leq k .
$$

We remark that theoretically for every bounded excess-demand function there exists a constant $k$ such that $\sum_{z_{i}\left(p^{*}\right)>0} z_{i}\left(p^{*}\right) \leq k$ for every $p$. But the purpose is to clarify how big or small this constant is for some $p^{*}$ in a given economy.

The constant $k \in \mathbf{R}_{+}$as a numerical value of the maximally possible unsatisfied demand for a given price $p^{*} \in S_{n}$ characterizes to what state the economy differs from the market equilibrium.

We can prove now the following

THEOREM 5.1. Let for some economy with a finite number $n$ of goods the Assumptions $F, H, D C$ and $\Gamma$ be fulfilled and put $w_{+}=\frac{-(n+1)+\sqrt{(n+1)^{2}+8 n \gamma}}{2 n}$. If $w<w_{+}$then there exists a $k$-equilibrium for each $k \geq \frac{n w^{2}+(n+1) w}{2 \gamma-n w^{2}-(n+1) w}$.

The number $w_{+}$is positive for each $n$ and fixed $\gamma>0$. If one takes $w=0$ then $k \geq 0$ and with $k=0$ there is obtained the classical equilibrium in means of Definition 2.1. We observe that in this case it is not necessary to use the Walras' Law for establishing an equilibrium. If one takes $w>0$ then we obtain the $k$-equilibrium with $k>0$.

Note that in the Arrow-Hahn models from Sections 2 and 3 it is impossible to carry out any quantitative analysis. Theorem 5.1 give a chance to analyse the behaviour of an economy for different numerical values of the parameters $n, w, \gamma$. If for fixed $n$ and $\gamma$ the value $w$ is sufficiently close to $w_{+}$then $k$ is very large.

\section{Economic models with weakened condition of $w$-discontinuous excess de-} mand functions. Our purpose in this section is to connect Arrow-Hahn model from Section 3 with model from Section 5. The description of model takes Arrow-Hahn model from Section 2 as a basis. We keep Assumptions $\mathrm{F}$ and $\mathrm{H}$ from Arrow-Hahn model and make changes in other assumptions. 
Assumption DC'. The excess-demand function $z$ is defined for every $p \in S_{n}$ such that $p_{i}>0, i=1,2, \ldots, n$, and possibly for other $p$ and is $w$-discontinuous whenever defined. If $z$ is not defined for $p^{0}$ then

$$
\lim _{p \rightarrow p^{0}} z_{i}(p)=\left\{\begin{array}{ll}
+\infty, & \text { if } p_{i}^{0}=0 \\
\in \mathbf{R}, & \text { if } p_{i}^{0} \neq 0
\end{array}, i=1,2, \ldots, n .\right.
$$

Since $z$ is not defined in the whole set $S_{n}$ then a small correction is necessary in Assumptions B and $\Gamma$.

Let $S_{n}^{\prime}=\left\{p \in S_{n} \mid z(p)\right.$ is defined $\}$.

Assumption B'. There exists a positive finite number $B$ such that for all $p \in S_{n}^{\prime}$ : $z_{i}(p)>-B$ for all $i=1,2, \ldots, n$, i.e., $z(p)$ is bounded from below in the set $S_{n}^{\prime}$.

Assumption $\Gamma^{\prime} \cdot \gamma=\inf _{p \in S_{n}^{\prime}} \gamma_{p}>0$ where for every $p \in S_{n}^{\prime}: \gamma_{p}=\sum_{z_{i}(p) \leq 0} p_{i}$.

For the norm in the space $\mathbf{R}^{n}$ we use the previous norm

$$
\|x\|=\sum_{i=1}^{n}\left|x_{i}\right|, \quad x=\left(x_{1}, x_{2}, \ldots, x_{n}\right) \in \mathbf{R}^{n} .
$$

By these assumptions we can prove existence of quasi-equilibrium in the sense of Definition 5.1. We divide the proof in two parts. At first we prove the lemma of technical nature for which all assumptions are not needed.

We define a mapping $M: S_{n} \rightarrow[0 ; 1]$, where $M(p)=\left(m_{1}(p), m_{2}(p), \ldots, m_{n}(p)\right)$ and if $z$ is defined at $p$, then

$$
m_{i}(p)= \begin{cases}0, & z_{i}(p)<0 \\ z_{i}(p), & 0<z_{i}(p)<1 \\ 1, & z_{i}(p) \geq 1\end{cases}
$$

if $z$ is not defined at $p$, then

$$
m_{i}(p)= \begin{cases}0, & \lim _{p^{k} \rightarrow p} z_{i}\left(p^{k}\right) \leq 0 \\ \lim _{p^{k} \rightarrow p} z_{i}\left(p^{k}\right), & 0<\lim _{p^{k} \rightarrow p} z_{i}\left(p^{k}\right)<1, \\ 1, & \lim _{p^{k} \rightarrow p} z_{i}\left(p^{k}\right) \geq 1\end{cases}
$$

and the sequence $\left(p^{k}\right)_{k \in \mathbf{N}}$ consists of points from neighbourhood of $p$ where $z(p)$ is defined, $i=1,2, \ldots, n, \quad e=(1, \ldots, 1)$ denotes the vector of $\mathbf{R}^{n}$ with all components equal to 1 .

For every $p \in S_{n}$ we define

$$
T(p)=\frac{p+M(p)}{(p+M(p)) e} .
$$

Lemma 6.1. Let for some economy with a finite number $n$ of goods Assumptions $F, H$, $B$ ' and $D C$ ' be fulfilled. Then there exists a $\left(n w^{2}+w(n+1)\right)$-discontinuous mapping $T: S_{n} \rightarrow S_{n}$.

Proof. We will show that the mapping (6.2) is as required. Note that $\sum_{i=1}^{n} m_{i}(p)=$ $\sum_{m_{i}(p) \geq 0} m_{i}(p) \geq 0$. Therefore

$$
0<(p+M(p)) e=\sum_{i=1}^{n}\left(p_{i}+m_{i}(p)\right)=1+\sum_{i=1}^{n} m_{i}(p) .
$$


Then $0 \leq t_{i}(p)=\frac{p_{i}+m_{i}(p)}{1+\sum_{i=1}^{n} m_{i}(p)} \leq 1$ for every $i=1,2, \ldots, n$ and

$$
\sum_{i=1}^{n} t_{i}(p)=\frac{\sum_{i=1}^{n}\left(p_{i}+m_{i}(p)\right)}{1+\sum_{i=1}^{n} m_{i}(p)}=\frac{1+\sum_{i=1}^{n} m_{i}(p)}{1+\sum_{i=1}^{n} m_{i}(p)}=1 .
$$

Therefore $T(p): S_{n} \rightarrow S_{n}$.

The mapping $M(p)$ is $w$-discontinuous for every $p \in S_{n}$ where $z(p)$ is defined. If $z$ is not defined for vector $p^{0}$ then by definition of limit there exists a neighbourhood $U\left(p^{0}\right)$ of point $p^{0}$ where $z(p)$ is defined. The coordinates of $z(p)$ are less than 1 or greater or equal to 1 for every $p \in U\left(p^{0}\right)$. By Assumption DC': if $p_{i}=0$ then $\lim _{p^{k} \rightarrow p^{0}} z_{i}\left(p^{k}\right)=+\infty$ where the sequence $\left(p^{k}\right)_{k \in \mathbf{N}}$ consists of points from a neighbourhood of $p^{0}$ where $z$ is defined. By Assumption B' the mapping $z$ is bounded from below. Since the mapping $m_{i}(p)$ is $w$-discontinuous in $U\left(p^{0}\right)$ then by definition of $m_{i}$ at the point $p^{0}(6.1)$ follows that the mapping $m_{i}(p)$ is $w$-discontinuous at $p^{0}$ too. Therefore the mapping $M$ is also $w$-discontinuous in the whole set $S_{n}$.

The particular maps which the map $T$ consists of, possess the following properties: the identity map $I$ on $S_{n}$ is continuous, $M(p)$ is $w$-discontinuous then by Corollary 4.1 the map $I+M(p)=p+M(p)$ is $w$-discontinuous. Then by Corollary 4.3 the map $(p+M(p)) e$ is $w\|e\|$-discontinuous. Since $\|e\|=n$ then $(p+M(p)) e$ is $n w$-discontinuous. Since for every $p \in S_{n}(p+M(p)) e \in\left[1,+\infty\left[\right.\right.$ then the map $\frac{1}{(p+M(p)) e}$ is $\frac{n w}{1+\sum_{m_{i}(p)>0} m_{i}(p)}$-discontinuous by Corollary 4.4. Finally the mapping $T(p)=(p+M(p)) \cdot \frac{1}{(p+M(p)) e}$ by Proposition 4.2 is $w^{*}(p)$-discontinuous where

$$
\begin{gathered}
w^{*}(p)=\frac{n w^{2}}{1+\sum_{m_{i}(p)>0} m_{i}(p)}+\frac{w}{1+\sum_{m_{i}(p)>0} m_{i}(p)}+\frac{n w\|p+M(p)\|}{1+\sum_{m_{i}(p)>0} m_{i}(p)}= \\
=\frac{n w^{2}+w}{1+\sum_{m_{i}(p)>0} m_{i}(p)}+n w \leq n w^{2}+(n+1) w .
\end{gathered}
$$

Therefore the map $T$ is also $n w^{2}+(n+1) w$-discontinuous on the set $S_{n}$.

THEOREM 6.1. Let for some economy with a finite number $n$ of goods the Assumptions $F, H, B$ ', DC' and $\Gamma^{\prime}$ be fulfilled. Let

$$
w_{+}=\frac{\left.-(n+1)+\sqrt{(n+1)^{2}+4 \min \left\{\frac{n}{n+1}, \gamma\right.}\right\}}{2 n} .
$$

If $w<w_{+}$then there exists a $k$-equilibrium for each

$$
k \geq \min \left\{n-1, \frac{n w^{2}+(n+1) w}{2 \gamma-\left(n w^{2}+(n+1) w\right)}\right\} .
$$

Proof. By Lemma 6.1 there exists a mapping (6.2), $T: S_{n} \rightarrow S_{n}$, that is $n w^{2}+(n+1) w$ discontinuous in the set $S_{n}$. Since $S_{n}$ is a convex and compact subset in the normed vector space $\mathbf{R}^{n}$ we conclude by means of Theorem 4.2 that there exists a vector $p^{*} \in S_{n}$ satisfying the inequality

$$
\left\|T\left(p^{*}\right)-p^{*}\right\| \leq n w^{2}+(n+1) w
$$


We prove that this point is the one where $k$-equilibrium exists. Using the norm in $\mathbf{R}^{n}$ this yields

$$
\begin{aligned}
\left\|T\left(p^{*}\right)-p^{*}\right\| & =\left\|\frac{p^{*}+M\left(p^{*}\right)}{\left(p^{*}+M\left(p^{*}\right)\right) e}-p^{*}\right\|=\left\|\frac{p^{*}+M\left(p^{*}\right)-p^{*}-p^{*} \sum_{i=1}^{n} m_{i}\left(p^{*}\right)}{1+\sum_{i=1}^{n} m_{i}\left(p^{*}\right)}\right\| \\
& =\frac{\sum_{i=1}^{n}\left|m_{i}\left(p^{*}\right)-p_{i}^{*} \sum_{i=1}^{n} m_{i}\left(p^{*}\right)\right|}{1+\sum_{i=1}^{n} m_{i}\left(p^{*}\right)} \leq n w^{2}+(n+1) w .
\end{aligned}
$$

First we ascertain that inequality (6.3) is not true for price vectors for which the mapping $z$ is not defined. For these vectors $p^{*}$ by Assumption DC' at least one coordinate of $M\left(p^{*}\right)$ is 1 and the corresponding coordinate of the price vector is 0 ; we assume that it is $j$-coordinate.

Note that $0<1+\sum_{i=1}^{n} m_{i}\left(p^{*}\right) \leq 1+n$. Then from (6.3) it follows that

$$
1 \leq 1+\sum_{i=1, i \neq j}^{n}\left|m_{i}\left(p^{*}\right)-p_{i}^{*} \sum_{i=1}^{n} m_{i}\left(p^{*}\right)\right| \leq\left(n w^{2}+(n+1) w\right)(1+n),
$$

it is true that $1 \leq(1+n) n w^{2}+(1+n)^{2} w$. From the last inequality it follows that

$$
w \geq \frac{-(1+n)+\sqrt{(1+n)^{2}+\frac{4 n}{n+1}}}{2 n}=w_{1} .
$$

$w<w_{1}$ from the condition of theorem.

If the mapping $z$ is defined for $p^{*}$ and at least one coordinate of $M$ is 1 then $1 \leq$ $\sum_{i=1}^{n} m_{i}\left(p^{*}\right)$. On the other hand by Assumption $\Gamma^{\prime}$ there exists at least one nonpositive coordinate of $z\left(p^{*}\right)$, i.e., $\exists j \in 1, \ldots, n: z_{j}\left(p^{*}\right) \leq 0$ and therefore $m_{j}\left(p^{*}\right)=0$. From the last equality it follows that $\sum_{i=1}^{n} m_{i}\left(p^{*}\right) \leq n-1$. Therefore it is possible to write the inequality $(6.3)$ in the following way

$$
\sum_{i=1}^{n}\left|m_{i}\left(p^{*}\right)-p_{1}^{*} \sum_{i=1}^{n} m_{i}\left(p^{*}\right)\right| \leq\left(n w^{2}+(n+1) w\right)(1+(n-1)) .
$$

The left side of (6.4) is greater than

$$
\sum_{z_{i}\left(p^{*}\right) \leq 0}\left|m_{i}\left(p^{*}\right)-p_{i}^{*} \sum_{i=1}^{n} m_{i}\left(p^{*}\right)\right|=\sum_{z_{i}\left(p^{*}\right) \leq 0} p_{i}^{*} \sum_{i=1}^{n} m_{i}\left(p^{*}\right)=\sum_{i=1}^{n} m_{i}\left(p^{*}\right) \sum_{z_{i}\left(p^{*}\right) \leq 0} p_{i}^{*} .
$$

Since $\sum_{i=1}^{n} m_{i}\left(p^{*}\right) \geq 1$ and satisfies Assumption $\Gamma^{\prime}$ then

$$
\sum_{i=1}^{n} m_{i}\left(p^{*}\right) \sum_{z_{i}\left(p^{*}\right) \leq 0} p_{i}^{*} \geq \sum_{z_{i}\left(p^{*}\right) \leq 0} p_{i}^{*} \geq \gamma
$$

Therefore in place of inequality (6.4) we consider

$$
\gamma \leq n^{2} w^{2}+n(n+1) w
$$

From this last inequality it follows that

$$
w \geq \frac{-(n+1)+\sqrt{(1+n)^{2}+4 \gamma}}{2 n}=w_{2} .
$$

In order to avoid this situation we have assumed that $w<w_{2}$ in the conditions of theorem. 
Now we consider the situation that all coordinates of $z\left(p^{*}\right)$ are strictly less than 1 . Then the numerator of the left side of inequality (6.3) can be split into two sums

$$
\begin{aligned}
\sum_{z_{i}\left(p^{*}\right) \leq 0}\left|0-p_{i}^{*} \sum_{i=1}^{n} m_{i}\left(p^{*}\right)\right| & +\sum_{0<z_{i}\left(p^{*}\right)<1}\left|z_{i}\left(p^{*}\right)-p_{i}^{*} \sum_{i=1}^{n} m_{i}\left(p^{*}\right)\right| \\
& =\sum_{i=1}^{n} m_{i}\left(p^{*}\right) \sum_{z_{i}\left(p^{*}\right) \leq 0} p_{i}^{*}+\sum_{0<z_{i}\left(p^{*}\right)<1}\left|z_{i}\left(p^{*}\right)-p_{i}^{*} \sum_{i=1}^{n} m_{i}\left(p^{*}\right)\right| .
\end{aligned}
$$

By using the triangle inequality we get the estimation

$$
\sum_{0<z_{i}\left(p^{*}\right)<1}\left|z_{i}\left(p^{*}\right)-p_{i}^{*} \sum_{i=1}^{n} m_{i}\left(p^{*}\right)\right| \geq\left|\sum_{0<z_{i}\left(p^{*}\right)<1} z_{i}\left(p^{*}\right)-p_{i}^{*} \sum_{i=1}^{n} m_{i}\left(p^{*}\right)\right| .
$$

By Assumption $\Gamma^{\prime}$

$$
\sum_{i=1}^{n} m_{i}\left(p^{*}\right) \sum_{z_{i}\left(p^{*}\right) \leq 0} p_{i}^{*} \geq \gamma \sum_{i=1}^{n} m_{i}\left(p^{*}\right)
$$

By definition of $M\left(p^{*}\right)$ it follows that $\sum_{i=1}^{n} m_{i}\left(p^{*}\right)=\sum_{0<z_{i}\left(p^{*}\right)<0} z_{i}\left(p^{*}\right)$. Therefore we obtain

$$
\begin{aligned}
\sum_{i=1}^{n} m_{i}\left(p^{*}\right) & \sum_{z_{i}\left(p^{*}\right) \leq 0} p_{i}^{*}+\sum_{0<z_{i}\left(p^{*}\right)<0}\left|z_{i}\left(p^{*}\right)-p_{i}^{*} \sum_{i=1}^{n} m_{i}\left(p^{*}\right)\right| \\
& \geq \gamma \sum_{0<z_{i}\left(p^{*}\right)<1} z_{i}\left(p^{*}\right)+\left|\sum_{0<z_{i}\left(p^{*}\right)<1} z_{i}\left(p^{*}\right)\left(1-\sum_{0<z_{i}\left(p^{*}\right)<1} p_{i}^{*}\right)\right| \\
& =\gamma \sum_{0<z_{i}\left(p^{*}\right)<1} z_{i}\left(p^{*}\right)+\sum_{0<z_{i}\left(p^{*}\right)<1} z_{i}\left(p^{*}\right) \sum_{z_{i}\left(p^{*}\right) \leq 0} p_{i}^{*} \geq 2 \gamma \sum_{0<z_{i}\left(p^{*}\right)<1} z_{i}\left(p_{i}^{*}\right) .
\end{aligned}
$$

From last inequalities the inequality (6.3) can be transformed

$$
2 \gamma \sum_{0<z_{i}\left(p^{*}\right)<1} z_{i}\left(p_{i}^{*}\right) \leq\left(n w^{2}+(n+1) w\right)\left(1+\sum_{0<z_{i}\left(p^{*}\right)<1} z_{i}\left(p^{*}\right)\right) .
$$

The last inequality yields

$$
\sum_{0<z_{i}\left(p^{*}\right)<1} z_{i}\left(p^{*}\right) \leq \frac{n w^{2}+(n+1) w}{2 \gamma-\left(n w^{2}+(n+1) w\right)},
$$

i.e., $\sum_{0<z_{i}\left(p^{*}\right)<1} z_{i}\left(p^{*}\right) \leq k$, where $k$ satisfies $k \geq \frac{n w^{2}+(n+1) w}{2 \gamma-\left(n w^{2}+(n+1) w\right)}$. In order to have the number $2 \gamma-\left(n w^{2}+(n+1) w\right)$ positive

$$
w<\frac{-(1+n)+\sqrt{(1+n)^{2}+8 n \gamma}}{2 n}=w_{3} .
$$

From the conditions of the theorem $w<w_{3}$. From the definition of $M$ in this last case we have $\sum_{0<z_{i}\left(p^{*}\right)<1} z_{i}\left(p^{*}\right) \leq n-1$.

If all coordinates of $z\left(p^{*}\right)$ are negative then $0 \leq n w^{2}+(n+1) w$ which is not in contradiction with previous conclusions. 
7. Conclusions. First we give two simple examples of an economy with discontinuous excess demand function that illustrates Theorem 6.1 .

EXAMPLE 7.1. We consider the economy with 2 different goods and 2 economic agents. Let

$$
S_{2}=\left\{p=\left(p_{1}, p_{2}\right) \mid p_{1}, p_{2} \geq 0 \text { and } p_{1}+p_{2}=1\right\}
$$

be a price set. In Figure 7.1 the values of $p=\left(p_{1}, p_{2}\right)$ are plotted on the horizontal axis (the origin is point $p^{\prime}=(0,1)$ and farther right is plotted point $p^{\prime \prime}=(1,0)$ ) and values of $z(p)$ coordinates are plotted on the vertical axis. We assume that excess demand function $z(p)=\left(z_{1}(p), z_{2}(p)\right)$ is

$$
\begin{aligned}
& z_{1}(p)= \begin{cases}\frac{3 p_{2}}{40 p_{1}}, & 0<p_{1} \leq \frac{1}{2}, p_{2}=1-p_{1} \\
0, & \frac{1}{2}<p_{1} \leq 1, p_{2}=1-p_{1}\end{cases} \\
& z_{2}(p)= \begin{cases}-\frac{3}{80}, & 0 \leq p_{1} \leq \frac{1}{2}, p_{2}=1-p_{1} \\
\frac{3 p_{1}}{80 p_{2}}, & \frac{1}{2}<p_{1}<1, p_{2}=1-p_{1}\end{cases}
\end{aligned}
$$

This function is homogeneous (Assumption $\mathrm{H}$ ):

$$
\begin{gathered}
z(\lambda p)=z\left(\left(\lambda p_{1}, \lambda p_{2}\right)\right)=\left(\begin{array}{c}
\frac{3 \lambda p_{2}}{40 \lambda p_{1}} \\
-\frac{3}{80}
\end{array}\right)=\left(\begin{array}{c}
\frac{3 p_{2}}{40 p_{1}} \\
-\frac{3}{80}
\end{array}\right)=z(p) \text { if } 0<p_{1} \leq \frac{1}{2} \text { and } \\
z(\lambda p)=\left(\begin{array}{c}
0 \\
\frac{3 \lambda p_{1}}{80 \lambda p_{2}}
\end{array}\right)=\left(\begin{array}{c}
0 \\
\frac{3 p_{1}}{80 p_{2}}
\end{array}\right)=z(p) \text { if } \frac{1}{2}<p_{1}<1 .
\end{gathered}
$$

The constant $w$ is $w=\frac{3}{40}$. We illustrate this situation in Figure 7.1.

It is clear that there is no $p \in S_{2}$ which satisfies the inequality $z(p)=\left(z_{1}(p), z_{2}(p)\right) \leq$ 0 . Assumptions F, H, B' and DC' are fulfilled. Assumption $\Gamma^{\prime}$ also holds (but not Walras' Law). Indeed, represent $p=\left(p_{1}, p_{2}\right) \in S_{2}$ as

$$
\left.p=(1-t) p^{\prime}+t p^{\prime \prime}, \quad t \in\right] 0,1[,
$$

then $\left.t \in] 0, \frac{1}{2}\right]$ implies $z_{1}(p)>0, z_{2}(p)<0$ and so $\gamma_{p}=p_{2}$ and $\left.t \in\right] \frac{1}{2}, 1\left[\operatorname{implies} z_{1}(p)=0\right.$, $z_{2}(p)>0$ and so $\gamma_{p}=p_{1}$. In both cases we get $\gamma_{p} \geq \frac{1}{2}$ which shows that Assumption $\Gamma^{\prime}$ holds with $\gamma=\frac{1}{2}$. Theorem 6.1 guarantees the existence of k-equilibrium for $k \geq$ $\min \left\{1 ; \frac{2 w^{2}+3 w}{1-2 w^{2}-3 w}\right\}$ if

$$
w<\frac{-3+\sqrt{9+4 \min \left\{\frac{2}{3} ; \frac{1}{2}\right\}}}{4}=\frac{-3+\sqrt{11}}{4}>\frac{-3+3.3}{4}=\frac{3}{40} .
$$

We have assumed that $w=\frac{3}{40}$ therefore $k \geq \min \left\{1 ; \frac{189}{611}\right\}=\frac{189}{611}$. Simple arithmetic shows that every

$$
p \in\left\{p=\left(p_{1}, p_{2}\right) \mid \frac{611}{3131} \leq p_{1} \leq \frac{5040}{5651}, p_{2}=1-p_{1}\right\}
$$

gives this $\frac{189}{611}$-equilibrium.

EXAmPle 7.2. Again we consider the economy with 2 different goods and 2 economic agents. Let

$$
S_{2}=\left\{p=\left(p_{1}, p_{2}\right) \mid p_{1}, p_{2} \geq 0 \text { and } p_{1}+p_{2}=1\right\}
$$




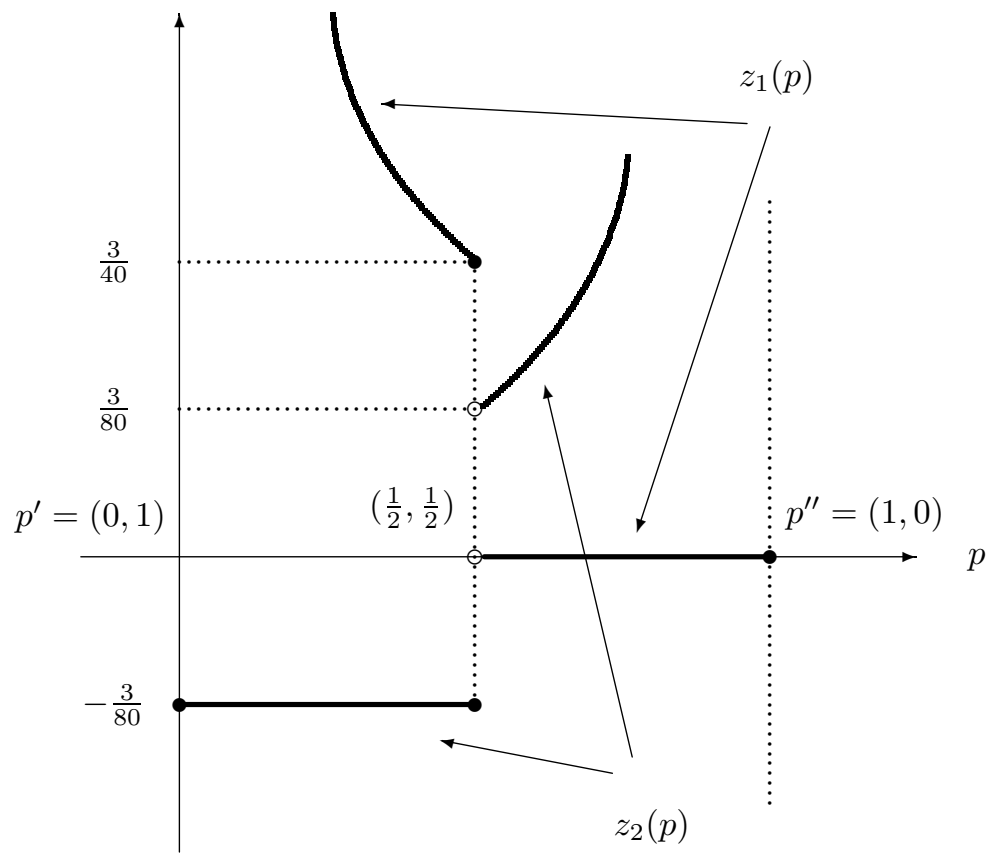

Fig. 7.1

be the price set. We assume that the excess demand function $z(p)=\left(z_{1}(p), z_{2}(p)\right)$ is

$$
\begin{aligned}
& z_{1}(p)= \begin{cases}\frac{1}{20}, & 0 \leq p_{1}<\frac{1}{2}, p_{2}=1-p_{1} \\
-\frac{p_{2}}{40 p_{1}}, & \frac{1}{2} \leq p_{1} \leq 1, p_{2}=1-p_{1}\end{cases} \\
& z_{2}(p)= \begin{cases}-\frac{p_{1}}{20 p_{2}}, & 0 \leq p_{1}<\frac{1}{2}, p_{2}=1-p_{1} \\
\frac{1}{40}, & \frac{1}{2} \leq p_{1} \leq 1, p_{2}=1-p_{1}\end{cases}
\end{aligned}
$$

This function is homogeneous (Assumption $\mathrm{H}$ ). The constant $w$ is similar as in Example $7.1\left(w=\frac{3}{40}\right)$. We illustrate this situation in Figure 7.2.

Assumptions F, H, B' and DC' are fulfilled. The similar arguments as in Example 7.1 show that Assumption $\Gamma^{\prime}$ holds with $\gamma=\frac{1}{2}$. But in this situation Walras' Law holds too:

$$
\begin{aligned}
& z_{1} p_{1}+z_{2} p_{2}=\frac{1}{20} p_{1}-\frac{p_{1}}{20 p_{2}} p_{2}=\frac{p_{1}}{20}-\frac{p_{1}}{20}=0 \text { if } 0 \leq p_{1}<\frac{1}{2}, p_{2}=1-p_{1} \text { and } \\
& z_{1} p_{1}+z_{2} p_{2}=-\frac{p_{2}}{40 p_{1}} p_{1}+\frac{1}{40} p_{2}=-\frac{p_{2}}{40}+\frac{p_{2}}{40}=0 \text { if } \frac{1}{2} \leq p_{1} \leq 1, p_{2}=1-p_{1}
\end{aligned}
$$

It is clear that there is no $p \in S_{2}$ which satisfies the inequality $z(p)=\left(z_{1}(p), z_{2}(p)\right) \leq 0$.

Theorem 6.1 guarantees the existence of $k$-equilibrium for $k \geq \min \left\{1 ; \frac{2 w^{2}+3 w}{1-2 w^{2}-3 w}\right\}$ if $w<\frac{-3+\sqrt{9+4 \min \left\{\frac{2}{3} ; \frac{1}{2}\right\}}}{4}=\frac{-3+\sqrt{11}}{4}>\frac{-3+3.3}{4}=\frac{3}{40}$. We have assumed that $w=\frac{3}{40}$ therefore $k \geq \min \left\{1 ; \frac{189}{611}\right\}=\frac{189}{611}$. Since $\frac{1}{40}<\frac{1}{20}<\frac{189}{611} \approx 0.309$ then we conclude that in this example every $p$ is $\frac{189}{611}$-equilibrium.

In this example also Assumptions F, H, DC and $\Gamma$ are fulfilled. Therefore Theorem 


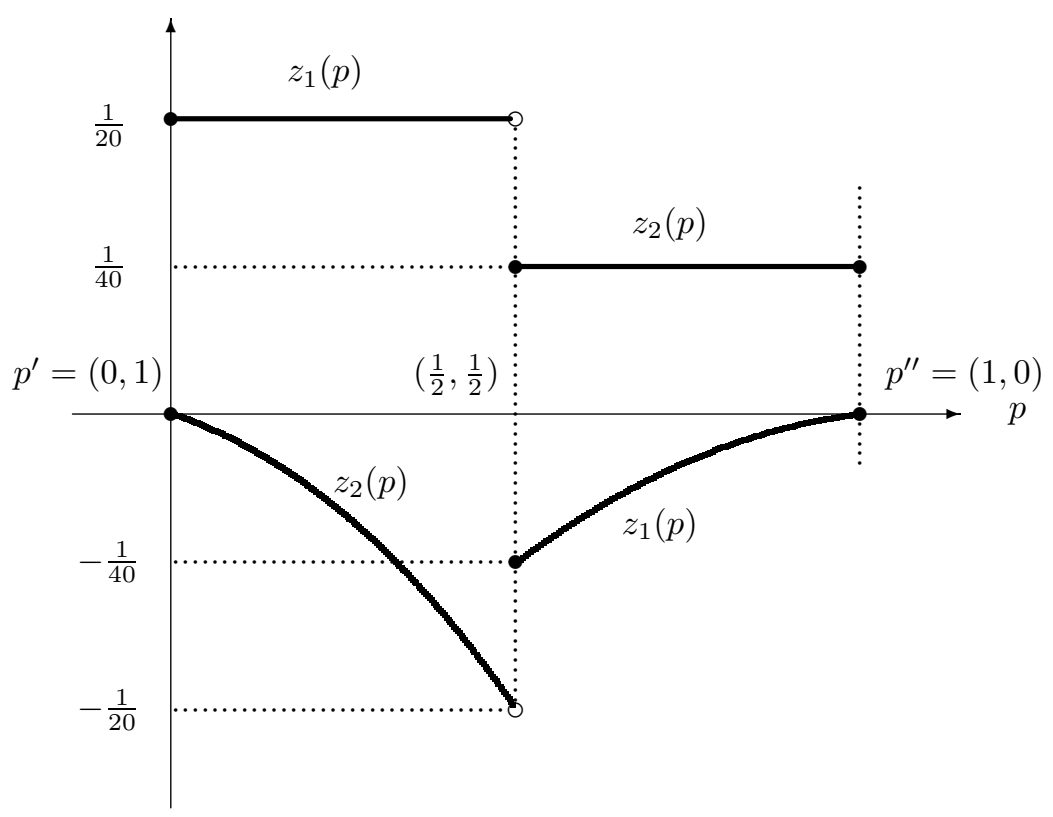

Fig. 7.2

5.1 guarantees the existence of $k$-equilibrium with every

$$
w<\frac{-3+\sqrt{9+8}}{4}=\frac{-3+\sqrt{17}}{4}>\frac{-3+4.1}{4}=\frac{11}{40} .
$$

We have assumed that $w=\frac{3}{40}$ therefore

$$
k \geq \frac{2 \cdot\left(\frac{3}{40}\right)^{2}+3 \cdot \frac{3}{40}}{1-2 \cdot\left(\frac{3}{40}\right)^{2}-3 \cdot \frac{3}{40}}=\frac{189}{611} .
$$

Every $p$ gives this $\frac{189}{611}$-equilibrium. We see that $k=\frac{189}{611}$ is rough estimation. Every $p \in\left\{p=\left(p_{1}, p_{2}\right) \mid \frac{1}{2} \leq p_{1} \leq 1, p_{2}=1-p_{1}\right\}$ is $\frac{1}{40}$-equilibrium.

\section{Remarks and conclusions}

1. The proof of Theorem 6.1 shows that we can estimate quasi-equilibrium only in the case $w<w_{+}$.

2. If $w=0$ (excess demand function is continuous in the set $S_{n}^{\prime}$ ) then we obtain by our assumptions the classical equilibrium. If $w>0$ then we obtain the $k$-equilibrium with $k>0$.

We should like to ask the economists to consider what is the meaning of $w=0.001$ or $w=10^{-10}$ ?

3. Examples 7.1 and 7.2 show that the value of the constant $k$ may be smaller than the bound in Theorems 5.1 and 6.1 .

4. In the definition of the function $M$ (see before Lemma 6.1) we use the constant 1 if $z_{i}(p) \geq 1$ or $\lim _{p^{k} \rightarrow p} z_{i}\left(p^{k}\right) \geq 1$. It is possible to choose another constant greater than 0 . Then we also get another constant $k$ for quasi-equilibrium. 
5. If $n \rightarrow \infty$ then $\min \left\{\frac{n}{n+1}, \gamma\right\} \rightarrow \min \{1, \gamma\}=\gamma$. We think that the constant $\gamma$ also is small number; also we think that always $\min \left\{\frac{n}{n+1}, \gamma\right\}=\gamma$.

6. The conclusion from Theorem 6.1 is almost the same as from Theorem 5.1 with the major difference of having bounded constant $k$ for every fixed $n$. Therefore this last model emphasizes that the constant $n$ (the number of goods) is an important economic parameter.

Acknowledgments. The authors are indebted to the referee and editors for their constructive remarks and patience.

\section{References}

[1] C. D. Aliprantis, D. J. Brown and O. Burkinshaw, Existence and Optimality of Competitve Equilibria, Springer-Verlag, Berlin, Heidelberg, New York, 1990.

[2] K. J. Arrow and G. Debreu, Existence of equilibrium for a competitive economy, Econometrica 42 (1954), 265-290.

[3] K. J. Arrow and F. H. Hahn, General Competitive Analysis, Advanced Textbooks in Economics 12, North-Holland, 1991.

[4] I. Bula, On the stability of Bohl-Brouwer-Schauder theorem, Nonlinear Analysis 26 (1996), 1859-1868.

[5] I. Bula, Approximating certain type of discontinuous mappings with continuous mappings, Int. J. of Applied Mathematics 2 (2000), 291-301.

[6] I. Bula and M. R. Weber, On discontinuous functions and their application to equilibria in some economic model, preprint of Technische Universität Dresden MATH-AN-02-02, 2002, 20 p.

[7] M. Burgin and A. Schostak, Towards the theory of continuity defect and continuity measure for mappings of metric spaces, Latvijas Universitātes Zinātniskie Raksti, Matemātika 576 (1992), 45-62.

[8] R. R. Cornwall, Introduction to the Use of General Equilibrium Analysis, Advanced Textbooks in Economics 20, North-Holland, 1984.

[9] G. Debre, Theory of Value: An Axiomatic Analysis of Economic Equilibrium, Yale University Press, New Haven and London, 1959.

[10] J. Dugundji and A. Granas, Fixed Point Theory, Springer Monographs in Mathematics, Springer-Verlag, New York, 2003.

[11] W. Hildenbrand and A. P. Kirman, Equilibrium Analysis, Advanced Textbooks in Economics 28, North-Holland, 1991.

[12] S. Lang, Analysis I, Addison-Wesley, 1976.

[13] D. M. Kreps, A Course in Microecnomic Theory, Harvester Wheatsheaf, 1990.

[14] K. Kuratowski, Topology I, Academic Press, New York, 1966.

[15] W. Nicholson, Microeconomic Theory. Basic Principles and Extensions, The Dryden Press, 1992.

[16] H. R. Varian, Mikroökonomie, R. Oldenburg Verlag, 1990.

[17] L. Walras, Elements of Pure Economics, Allen and Unwin, London, 1954.

[18] O. Zaytsev, On discontinuous mappings in metric spaces, Proc. of the Latvian Academy of Sciences, Section B, 52 (1998), 259-262. 